\title{
Using Similarity-based Operations for Resolving Data-level Conflicts
}

\author{
Eike Schallehn and Kai-Uwe Sattler \\ Department of Computer Science, University of Magdeburg, \\ P.O. Box 4120, D-39106 Magdeburg, Germany \\ \{eike|kus\}@iti.cs.uni-magdeburg.de
}

\begin{abstract}
Dealing with discrepancies in data is still a big challenge in data integration systems. The problem occurs both during eliminating duplicates from semantic overlapping sources as well as during combining complementary data from different sources. Though using SQL operations like grouping and join seems to be a viable way, they fail if the attribute values of the potential duplicates or related tuples are not equal but only similar by certain criteria. As a solution to this problem, we present in this paper similarity-based variants of grouping and join operators. The extended grouping operator produces groups of similar tuples, the extended join combines tuples satisfying a given similarity condition. We describe the semantics of these operators, discuss efficient implementations for the edit distance similarity and present evaluation results. Finally, we give examples how the operators can be used in given application scenarios.
\end{abstract}

\section{Introduction}

In the past few years, there has been a great amount of work on data integration. This includes the integration of information from diverse sources in the Internet, the integration of enterprise data in support of decision-making using data warehouses, and preparing data from various sources for data mining. Some of the major problems in this context - besides overcoming structural conflicts - are related to overcoming conflicts and inconsistencies on the data level. This includes the elimination of duplicate data objects caused by semantic overlapping of some sources, as well as establishing a relationship between complementary data from these sources. The implementation of associated operations has a significant difference to usual data management operations: only in some rare cases can we rely on equality of attributes. Instead we have to deal with discrepancies in data objects representing the same or related real-world objects which may exist due to input errors or simply due to the autonomy of the sources. Furthermore, the amount of data to be processed in integration scenarios can be equal to, or even greater than that from a single source, so, efficiency of the implementation becomes a critical issue.

Duplicate elimination is a sub-task of data cleaning that comprises further tasks for improving data quality like transformation, outlier detection etc. Assuming SQL-based integration systems, the natural choice for duplicate elimination is the group by operator using the key attributes of the tuples in combination with aggregate functions for reconciling divergent non-key attribute values. However, this approach is limited to 
equality of the key attributes - if no unique key exists or the keys contain differences, tuples representing the same real-world object will be assigned to different groups and cannot be identified as equivalent tuples. The same is true for linking complementary data, which in a SQL system would be done based on equality by the join operator.

In this paper we address these problems and present similarity-based operators for joining and grouping based on previous work. We extend our earlier work by giving clear semantics of the operators, describing the implementation and evaluating optimization techniques. Both operators are based on extended concepts for similaritybased predicates. Major concerns are the new requirements resulting from the characteristics of similarity relationships, most of all atransitivity, and support for the efficient processing of similarity predicates.

The operators have not necessarily to be provided as a language extension, though we did this in our own query engine and use this syntax for illustration purposes. Instead it also can be implemented by utilizing extension mechanisms which are offered by today's DBMS. The implementation and the evaluation results described in this paper are based on table functions available in Oracle8i.

The remainder of this paper is organized as follows. After a discussion of related work in Section 2, we describe the characteristics and requirements of similarity predicates useful in data integration in Section 3. The proposed similarity operators are defined with respect to their semantics in Section 4. In Section 5 we describe strategies for an efficient implementation of these operators focusing on edit distances similarity measures. Results of our evaluation are given in Section 6. Finally, in Section 7 we present several aspects of the application of the similarity operations. Section 8 concludes the paper and points out ongoing work.

\section{Related Work}

The concepts described in this paper are intended to be used in data integration and cleaning scenarios. Related topics are from this field and similarity-based data operations, as well as from the field of analytical data processing.

Closely related to similarity-based operations is the integration of probabilistic concepts in data management. In [3] Dey et. al. propose an extended relational model and algebra supporting probabilistic aspects. Fuhr describes a probabilistic Datalog in [6]. Especially, for data integration issues and the aforementioned problems probabilistic approaches were verified and yielded useful results, as described by Tseng et. al. in [22]. The WHIRL system and language described in [2] by Cohen is based on Fuhr's work and uses text-based similarity and logic-based data access as known from Datalog to integrate data from heterogeneous sources. Cohen describes an efficient algorithm to compute the top scoring matches of a ranked result set. The implementation of the similarity predicate uses inverted indexes common in the field of information retrieval. A general framework for similarity joins for predicates on data types that can be mapped to multi-dimensional spaces is presented by Shim et. al. in [21]. The approach is based on an extended version of the $\mathrm{kdB}$ tree.

While efficient implementations of similarity predicates can be provided based on established index structures described above, most of the real-life applications consid- 
ered in this paper require predicates for string attributes. Though there is a number of similarity measures for strings, namely the edit distance and it's derivatives, for which a good overview is given by Navarro in [18], the efficient implementation for large data sets is a current research topic. In [9] Gravano et. al. present an approach for similaritybased joins on string attributes using an efficient pre-selection of $q$-grams for optimization. In short, the approach is based on down-sizing the data sets on which a similarity predicate is evaluated by first doing an equality-based join on substrings of fixed length $q$. Though our approach is not limited to string based predicates, we implemented an edit distance string similarity predicate using a trie as an index structure based on results by Shang and Merret described in [20] for evaluation purposes.

A major focus of our work is the problem of duplicate detection. This problem was discussed extensively in various research areas like database and information system integration [25, 14], data cleaning [1,7], information dissemination [24], and others. Early approaches were merely based on the equality of attribute values or derived values. Newer research results deal with advanced requirements of real-life systems, where identification very often is only possible based on similarity. Those approaches include special algorithms $[16,11]$, the application of methods known from the area of data mining and even machine learning [13].

An overview of problems related to entity identification is given in [12]. In [14] Lim et. al. describe an equality based approach, include an overview of other approaches and list requirements for the entity identification process. Monge and Elkan describe an efficient algorithm that identifies similar tuples based on a distance measure and builds transitive clusters in [17]. In [7] Galhardas et. al. propose a framework for data cleaning as a SQL extension and macro-operators to support among other data cleaning issues duplicate elimination by similarity-based clustering. The similarity relationship is expressed by language constructs, and furthermore, clustering strategies to deal with transitivity conflicts are proposed. Luján-Mora and Palomar propose a centroid method for clustering in [15]. Furthermore, they describe common discrepancies in string representations and derive a useful set of pre-processing steps and extended distance measures combining edit distance on a token-level and similarity of token sets. In [11] Hernández et. al. propose the sliding window approach for similarity-based duplicate identification where a neighborhood conserving key can be derived and describe efficient implementations.

The importance of extended concepts for grouping and aggregation in information integration is emphasized by Hellerstein et. al. in [10]. In particular, user-defined aggregation (UDA) were proposed in SQL3 and are now supported by several commercial database systems, e.g. Oracle, IBM DB2, Informix. In [23] the SQL-AG system for specifying UDA is presented, that translates to $C$ code. A more recent version of this approach called AXL is described in [23] and its usage in data mining is discussed.

\section{Similarity Measures}

Similarity based operators like the similarity join and the similarity-based grouping discussed here are based on similarity measures for attribute values and their logical combination. Other operators requiring concepts of similarity include for instance nearest 
neighbour queries and attribute similarity selections. These concepts currently find their way into commercial data management solutions, or are the topic of ongoing research. This section discusses useful similarity measures, their characteristics and requirements for common applications.

\subsection{Basic Similarity Predicates}

We use the following basic terms of similarity measures: let $x$ and $y$ be objects in a given universe of discourse $U$, a similarity measure is a function $\operatorname{sim}(x, y) \rightarrow[0,1]$. Alternatively a distance measure $d(x, y) \rightarrow \mathbb{R}$ can be used. The latter can be transformed to a similarity measure, for instance using the simple transformation $\operatorname{sim}(x, y)=1-$ $\frac{d(x, y)}{\max }$, where max is the maximum difference between objects in $U$, if applicable. This transformation implies a normalization, though other normalizations of distances within a given range are conceivable. A binary similarity predicate $\operatorname{SIM}(x, y) \subseteq U^{2}$, meaning " $y$ is similar to $x$ ", can for instance be derived from a similarity or distance measure using thresholds $t \in[0,1]$ or $k \in \mathbb{R}$ like $\operatorname{SIM}(x, y) \Leftrightarrow \operatorname{sim}(x, y) \geq t$ or $\operatorname{SIM}(x, y) \Leftrightarrow d(x, y) \leq k$. $S I M$ is in most cases considered as a reflexive, symmetric and atransitive relation.

While a number of approaches to describe similarity stemming from areas like information retrieval, multimedia data management or case-based reasoning exist, one of the major problems of expressing similarity within sets of structured data is, that the concept of similarity is in most cases highly dependent on the given application domain. Therefore, we describe basic similarity measures for common data types and ways of using these as primitives for combination to derive measures suitable for real life applications.

A widely used measure is the distance $d$ of data points $x, y$ in a metric space $S$, for instance the Euclidean Distance in an $n$-dimensional space. In a metric space the distance function fulfills the following conditions:

$$
\begin{gathered}
\forall x, y \in S \quad d(x, y)=0 \Leftrightarrow x=y \\
\forall x, y \in S d(x, y)=d(y, x) \\
\forall x, y, z \in S d(x, y) \leq d(x, z)+d(z, y)
\end{gathered}
$$

Especially the symmetry and the triangular inequality of such a distance measure given in (2) and (3) provide the fundament for efficient applications, e.g. in information retrieval and data mining. To use such measures, the data objects to be compared solely consist of coordinates in a metric space, or otherwise have to be transformed to represent points in this space, e.g. extracting feature vectors from multimedia data or deriving term-based vector representations of textual data. Supported by multi-dimensional indexing, predicates on these distance measures can be used efficiently, though efficiency is limited by the number of dimensions.

Another well-studied distance measure is the Levenshtein or edit distance edist $(p, w)$ on string representations. Certain costs are assigned to operations like insertion, deletion or substitution of characters to transform an original pattern string $p$ to a comparison string $w$, and the minimal distance is computed. For instance, assuming constant costs of 1 for the three mentioned basic operations, the edit distance of "edna" 
and "eden" is 2 , because the smallest sets of applicable operations are substitute(\#3, "e"), substitute(\#4," $n ")\}$ and $\{$ insert(\#3,"e"), delete(\#5)\} both having two operations. Common derivates also allow a transposition operation or apply heuristic-based costs for the operations, e.g. substituting or deleting vowels is usually less expensive than operations on consonants. This distance measure fulfills the three conditions given above for distances in metric space, this way granting efficient implementations. Though the edit distance is a powerful measure to detect inconsistencies in data, for instance for applications in the field of data integration and data cleaning, it is not widely used in current data management solutions. In Sections 5 and 6 we present an efficient implementation of a similarity predicate based on edit distance used with index-based optimization through tries as proposed in [20]. Other distance measures for strings include the Hamming distance, allowing only substitutions, the episode distance, allowing only insertions, and the longest common subsequence distance allowing insertions and deletions. A good overview of approximate string matching is given in [18]. Similar concepts of edit distances exist for other types of data representations, e.g. special sequences like genome data, spatio-temporal data, trees and graphs in general.

Textual and numerical data, the latter including the special case of 1-dimensional data and the difference as a distance measure plus widely used index structures like B-trees, is covered by the approaches introduced so far. A similarity measure for categorical data can be defined, if the categories can be mapped to a simple partial order, a metric space as described above, or a graph representing categories and their relationships. Distance measures for nodes in graphs are not discussed here, but it is worth mentioning that for graphs, as well as for sets, meaningful distance measures can be defined, that do not fulfill the criteria of symmetry and the triangular inequality.

\subsection{Complex and Application-specific Similarity}

So far we have discussed similarity measures applicable to atomic or homogeneously structured data types independently of a special application scenario. In real-life scenarios the expression of similarity has to deal with additional aspects to improve efficiency and the results of similarity based operations.

Complex similarity conditions: Similarity-based operators have to process tuples or more complex objects. The description of similarity between two of those objects may consist of a combination of more than one similarity predicate for an attribute and may use different similarity measures on them, e.g., for information on paintings in a database we can use the edit distance on artist names and the distance of vector representations for descriptions of the pictures contents.

Application-specific similarity measures: The semantics of values to be compared in given applications is known, which allows the usage of more precise similarity measures based on domain knowledge. Though we could use the edit distance to compare names of persons, we achieve better results if the similarity measure would consider that "Andy Warhol", "A. Warhol" and "Warhol, Andy" most likely refer to the same person.

By using similarity predicates as described above we can simply build complex similarity conditions by applying the logical operators $\wedge, \vee$ and $\neg$. As an alternative, a fuzzy logic can be applied to similarity measures directly, as proposed for instance in [2]. To 
reach the level of expressiveness we gain by specifying thresholds as part of every similarity predicate in the former approach, the concept of weighting the desired impact of every similarity measure would have to be added to the latter. An efficient evaluation of a complex similarity condition consisting of similarity predicates is described in Section 5.

Application-specific similarity measures and predicates can be defined in terms of user-defined functions as supported in most database systems. As an example consider a function $\operatorname{distName}(x, y)$ that takes into account the various conventions for writing names as described above. The algorithm can remove special characters, tokenize the string, find first letter matches and finally apply edist(token 1 ,token2) on candidate tokens, that possibly represent the last name, to take care of typos or inconsistent spelling of names.

Efficiency, one of the major problems of user-defined similarity, is discussed more detailed in Section 5. The general strategy would be to conjunctively combine the userdefined similarity predicates with index-supported equality or similarity predicates for pre-selection purposes. Asymmetric similarity measures are not considered here, so symmetry remains a requirement that has to be granted by the user-defined measure.

Existing operations in the relational algebra base largely on equivalence relations established through the equality of attribute values. To integrate with these concepts an equivalence relation can be derived from an atransitive similarity predicate SIM. Because establishing this equivalence relation is not our major focus here, throughout this paper we use the simple strategy of constructing an equivalence relation $S_{E Q}$ by building the transitive closure $S I M_{E Q}:=S I M^{+}$, i.e. a partition of the universe of discourse $U$ is a maximal set of objects that are similar either directly or indirectly. Especially related to entity identification, centroid or density-based clustering techniques proved to be useful strategies for dealing with atransitivity and provide a high level of accuracy, as for instance described in [15] and [17].

\section{Semantics of the Similarity Operators}

In this section we describe the semantics of our similarity-based operators as extensions of the standard relational algebra. We assume the following basic notations: let $R$ be a relation with the schema $S=\left\{A_{1}, \ldots, A_{m}\right\}, t^{R} \in R$ is a tuple from the relation $R$ and $t^{R}\left(A_{i}\right)$ denotes the value of attribute $A_{i}$ of the tuple $t^{R}$.

The core concept for similarity-based operations is a similarity condition. It expresses whether two tuples are similar in terms of their attribute values. Because we define our operators as an extension of the standard relational algebra, we do not deal with probabilities in conditions - by using a similarity threshold we can always rely on boolean values for such conditions. Hence, a similarity condition $<$ sim_cond $>$ is a conjunction of predicates:

$$
<\text { sim_cond }>=\bigwedge_{i=1}^{m}<\text { sim_pred }>\left(A_{i}\right)
$$


where < sim_pred $>$ denotes an atomic predicate which could be either eq or a "similarity predicate" like with an associated threshold or any other similarity predicate as discussed in Section 3.

Similarity join. Based on the similarity condition introduced above the semantics of the similarity join between two relations $R_{1}$ and $R_{2}$ can be described in a straightforward way. For a given similarity condition $<$ sim_cond $>$ we denote the set of all attributes referenced in this expression as

$$
\widetilde{S}=\left\{A_{i} \mid A_{i} \text { is referenced in }<\text { sim_cond }>\right\}
$$

and $S_{i}$ as the set of all attributes from relation $R_{i}$. Then, it holds

$$
\begin{aligned}
R_{1} \bowtie_{<\text {sim_cond }>} R_{2}=\{t \mid & \exists t_{1} \in R_{1}: t_{1}\left(S_{1}-\widetilde{S}\right)=t\left(S_{1}-\widetilde{S}\right) \wedge \\
& \exists t_{2} \in R_{2}: t_{1}\left(S_{2}-\widetilde{S}\right)=t\left(S_{2}-\widetilde{S}\right) \wedge \\
& \left.<\text { sim_cond }>\left(t_{1}, t_{2}\right)=\text { true }\right\}
\end{aligned}
$$

This simply means, a pair of tuples from the relations $R_{1}$ and $R_{2}$ appears in the result of the join operation if the similarity condition is fulfilled for these two tuples.

Similarity grouping. For defining the semantics of the grouping operator we rely on the algebra operator for standard grouping as presented in database textbooks [5]:

$$
<\text { grouping_attrs }>\mathcal{F}[<\text { aggr_func_list }>](R)
$$

Here <grouping_attrs > is a list of attributes used for grouping relation $R$, $<$ aggr_func_list> denotes a list of aggregate functions (e.g., count, avg, min, max etc.) conveyed by an attribute of relation $R$. For simplification, we assume that the name of an aggregated column is derived by concatenating the attribute name and the name of the function. An aggregate function $f$ is a function returning a value $v \in$ Dom for a multi-set of values $v_{1}, \ldots v_{m} \in$ Dom:

$$
f\left(\left\{v_{1}, \ldots, v_{m}\right\}\right)=v
$$

where Dom denotes an arbitrary domain of either numeric or alphanumeric values and the brackets $\{\ldots\}\}$ are used for multi-sets. We extend this equality-based grouping operator $\mathcal{F}$ with regard to the grouping criteria by allowing an similarity condition and call this new operator $\Gamma$ :

$$
<\text { sim_cond }>\Gamma[<\text { aggr_func_list }>](R)
$$

This operator again has a list of aggregate functions <aggr_func_list $>$ with the same meaning as above. However, the grouping criteria $<$ sim_cond $>$ is now a similarity conjunction as introduced above. The result of $\Gamma$ is a relation $R^{\prime}$ where the schema consists of all the attributes referenced in <sim_cond> accompanied with eq and the attributes named after the aggregates as described above. The relation $R^{\prime}$ is obtained by the concatenation of the two operators $\gamma$ and $\psi$ which reflect the two steps of grouping 
and aggregation. The first operator $\gamma_{<\text {sim_cond }>}(R)=\mathcal{G}$ produces a set of groups $\mathcal{G}=$ $\left\{G_{1}, \ldots, G_{m}\right\}$ from an input relation $R$. Each group is a non-empty set of tuples with the same schema as $R$. Furthermore, all tuples $t_{i}^{G}$ of a group $G$ are transitively similar to each other regarding the similarity condition $<$ sim_cond $>$ :

$$
\forall G \in \mathcal{G}: \forall t_{i}^{G}, t_{j}^{G} \in G: t_{j}^{G} \in t_{\operatorname{sim}}^{<\operatorname{sim} \_c o n d>}\left(t_{i}^{G}\right)
$$

where $\operatorname{sim}_{<\text {sim_cond }>}(t)$ denotes the set of all tuples which are in the transitive closure of the tuple $t$ with regard to sim_cond:

$$
\begin{aligned}
& t_{\text {sim }}^{<\text {sim_cond }>}(t)=\left\{t^{\prime} \mid \operatorname{sim} \_ \text {cond }\left(t, t^{\prime}\right)=\text { true } \vee\right. \\
& \left.\exists t^{\prime \prime} \in \operatorname{tsim}_{<\text {sim_cond }>}(t): \operatorname{sim} \_ \text {cond }\left(t^{\prime}, t^{\prime \prime}\right)=\text { true }\right\}
\end{aligned}
$$

and no tuple is similar to any other tuple of other groups

$$
\begin{array}{r}
\forall G_{i}, G_{j} \in \mathcal{G}, i \neq j: \forall t_{k}^{G_{i}} \in G_{i} \nexists t_{l}^{G_{j}} \in G_{j}: \\
\operatorname{sim\_ cond}\left(t_{k}^{G_{i}}, t_{l}^{G_{j}}\right)=\text { true }
\end{array}
$$

The second operator $\psi_{A_{1}, \ldots, A_{l},<\text { aggr_func_list }>}(\mathcal{G})=R^{\prime}$ reconciles (i.e., merges) the tuples from each group and produces exactly one tuple for each group of $\mathcal{G}$ according to the given aggregate functions. Thus, it holds $\forall G \in \mathcal{G}$ with $G=\left\{t_{1}^{G}, \ldots, t_{n}^{G}\right\}$ there is one and only one tuple $t^{R^{\prime}} \in R^{\prime}$ with

$$
\forall i=1 \ldots l: t^{R^{\prime}}\left(A_{i}\right)=t_{1}^{G}\left(A_{i}\right)=t_{2}^{G}\left(A_{i}\right)=\cdots=t_{n}^{G}\left(A_{i}\right)
$$

where $A_{1}, \ldots, A_{l}$ are attributes referred by the eq predicates of the approximation condition, (i.e., for these attributes all tuples have the same value) and

$$
\forall j=l+1 \ldots m-l: t^{R^{\prime}}\left(A_{j}\right)=f_{j-l}\left(\left\{t_{1}^{G}\left(A_{j}\right), \ldots, t_{n}^{G}\left(A_{j}\right)\right\}\right)
$$

where $f_{1}, \ldots, f_{m}$ are aggregate functions from <aggr_func_list $>$. Based on these two operators we can finally define the $\Gamma$ operator for similarity-based grouping as follows:

$$
<\text { sim_cond }>\Gamma[<\text { aggr_func_list }>](R)=\psi_{A_{1}, \ldots, A_{l},<\text { aggr_func_list }>}\left(\gamma_{<\text {sim_cond }>}(R)\right)
$$

where $A_{1}, \ldots, A_{l}$ are again attributes referenced by the eq predicates in $<$ sim_cond $>$.

\section{Implementation and Optimization}

In this section we outline our implementation of the similarity-based operators introduced in the previous sections. For an efficient realization dedicated plan operators are required, which implement the semantics described above. That means for instance for the similarity join, even if one formulates a query as follows

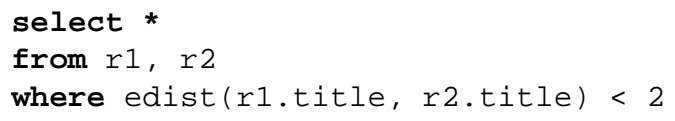


the similarity join implementation exploiting special index support has to be chosen by the query optimizer instead of computing the Cartesian product followed by a selection. In case of the similarity grouping a simple user-defined function is not sufficient as grouping function, because during similarity grouping the group membership is not determined by one or more of the tuple values but depends on already created groups. In addition, processing a tuple can be conveyed by merging existing groups.

Thus, we describe in the following the implementation of these two plan operators SIMJOIN and SIMGROUPING and assume, that the query optimizer is able to recognize the necessity of applying these operators during generating the query plan. This could be supported by appropriate query language extensions, e.g. for the similarity join like

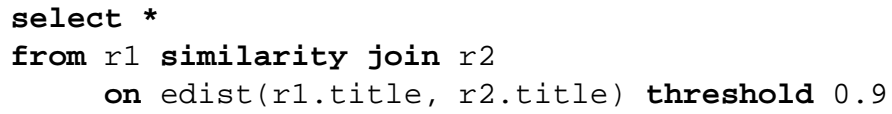

where threshold specifies the maximum allowed value for the normalized edit distance. For the similarity grouping this could be formulated as follows:

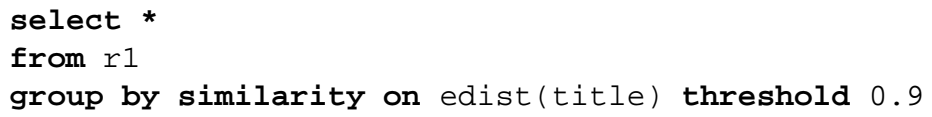

Currently, for our implementation we focus on edit distances as the primary similarity measure. For this purpose, we have adopted the approach proposed in [20] of using a trie in combination with a dynamic programming algorithm for computing the edit distance. The main idea is to traverse the trie containing the string values of all already processed tuples in depth-first order, trying to find a match with the search pattern, i.e., the attribute value of the currently processed tuple. Due to the usage of the edit distance, we must not stop the traversal directly after a found mismatch. Instead an edit operation (insert, remove or replace a character) is applied and the search is continued. Only after exceeding the given threshold, we can stop the traversal and go back to the next subtrie. Hence, the threshold is used for cutting off sub-tries containing strings not similar to the pattern. In addition, the effort for computing the dynamic programming tables required for determining the edit distance can be reduced, because all strings in one subtree share a common prefix and therefore the same edit distance. We omit further details of this algorithm and refer instead to the original work. In our implementation of the previously introduced operators tries are created on the fly for each grouping attribute or join predicate which appears together with an edit distance predicate.

\subsection{Similarity Join}

The implementation of a similarity join outlined in this section is quite straightforward, only differing in their usage of similarity predicates as join conditions. Like for conventional join operators index support for predicates can be exploited to improve performance by reducing the number of pairwise comparisons. However, the different predicates of a similarity expression require different kinds of index structures: 


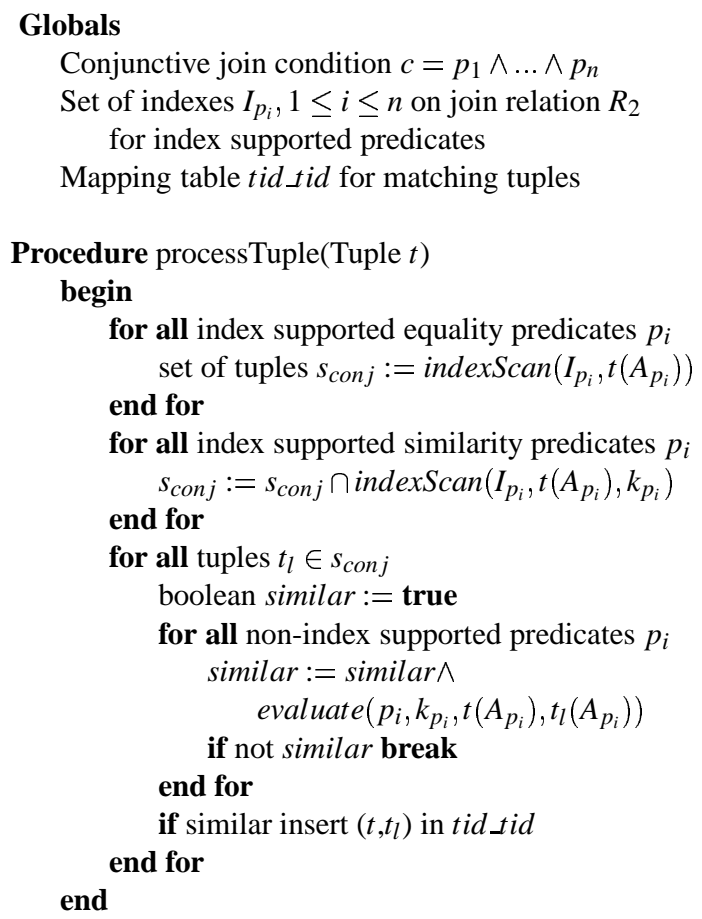

- For equality predicates eq $\left(A_{i}\right)$ common index structures like hash tables or B-trees can be utilized.

- Numeric approximation predicates like $\operatorname{diff}_{k}\left(A_{i}\right)$ can be easily supported by storing the minimum and maximum value of the attribute for each group.

- For string similarity based on edit distances edist $\left(A_{i}\right)$ tries are a viable index structure, as previously introduced.

- For the other similarity predicates discussed in Section 3 index support is given, for instance through multi-dimensional indexes like R-trees and its derivates on data mapped to a metric space.

Given such index structures a join algorithm can be implemented taking care of the various kinds of indexes. In Algorithm 1 a binary join for two relations $R_{1}$ and $R_{2}$ is shown, assuming that indexes for relation $R_{2}$ either exist or were built on the fly in a previous processing step. The result of this algorithm is a table of matching tuples for usage described later on. Alternatively, result tuples can be produced for pipelined query processing directly at this point. The notations $I_{p_{i}}$ and $k_{p_{i}}$ refer to the index on predicate $p_{i}$ and the specified threshold, respectively. $A_{p_{i}}$ refers to the involved attribute. 
As a side note, more complex similarity conditions could easily be supported by adding disjunctions. The similarity condition $c$ can be transformed to disjunctive normal form. For all conjunctions of $c=\bigvee_{i=1}^{m} \operatorname{conj} j_{i}$ the $s_{c o n j_{i}}$ are computed and the set of relevant groups would be $s_{d i s j}=\bigcup_{i=1}^{m} s_{c o n j_{i}}$.

\subsection{Similarity-based Grouping}

Like the join operator, the similarity-based grouping operator is based on the efficient evaluation of similarity predicates, but in addition has to deal with problems arising from the atransitivity of similarity relations. The goal of a grouping operator is to assign every tuple to a group. A naive implementation of the similarity-based operator would work as follows:

1. Iterate over the input set and process each tuple by evaluating the similarity condition with all previously processed tuples. Because these tuples were already assigned to groups, the result of this step is a set of groups.

2. If the result set is empty, a new group is created, otherwise the conflict is resolved by merging the groups according to the transitive closure strategy.

Other grouping strategies, like for instance density-based clustering, may in contrast require more rigid similarity relations between tuples in a group. In case of any conflict with a found group or between more than one found groups, existing groups would be split and maybe not considered during further processing. This behavior can be utilized to provide pipelined processing of the operator.

Obviously, the previously described naive implementation would lead to $O\left(n^{2}\right)$ time complexity for an input set of size $n$. Similar to processing a similarity join we assume that there are index-supported predicates for equality and similarity, and in addition, predicates like user-defined similarity predicates, that can not be supported by indexes. An according Algorithm was implemented and is described in detail in [19].

\subsection{Implementation using Oracle8i}

Implementing the described similarity operators in a SQL DBMS as native plan operators supporting the typical iterator interface [8] requires significant modifications to the database engine and therefore access to the source code. So, in order to add these operators to a commercial system the available programming interfaces and extensibility mechanisms should be used instead. Most modern DBMS support so-called table functions which can return tables of tuples, in some systems also in a pipelined fashion. In this way, our operators can be implemented as table functions consuming the tuples of a query, performing the appropriate similarity operation and returning the result table. For example, a table function sim_jo in implementing Algorithm 1 and expecting two cursor parameters for the input relations and the similarity join condition could be used as follows:

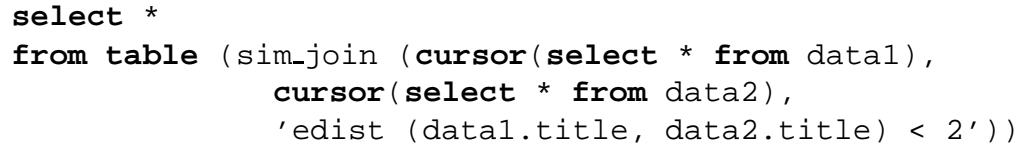


However, a problem of using table functions for implementing query operators are the strong typing restrictions: for the table functions a return type has always to be specified that prevents to use the same function for different input relations.

As one possible solution we have implemented table functions using and returning structures containing generic tuple identifiers (e.g., Oracle's rowid). So, the SIMGROUPING function produces a tuple of tuple identifier / group identifier pairs, where the group identifier is an artificial identifier generated by the operator. Based on this, the result type gid_tid_table of the table function is defined as follows:

create type gid_tid_t as object gid int, tid int);

create type gid_tid_table is table of gid_tid_t;

Using a grouping function sim_grouping a query can be written as the following query:

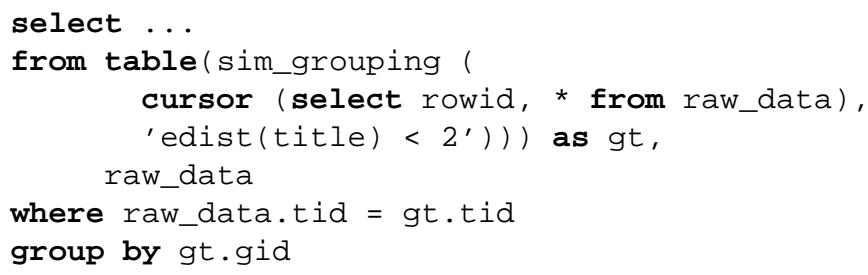

This approach allows to implement the function in a generic way, i.e., without any assumption on the input relation. In order to apply aggregation or reconciliation to the actual attribute values of the tuples, they are retrieved using a join with the original relation, whereas the grouping is performed based on the artificial group identifiers produced by the grouping operator.

In the same way, the SIMJOIN operator was implemented as a table functions returning pairs of tuple identifiers that fulfill the similarity condition and are used to join with the original data.

\section{Evaluation}

The similarity-based grouping and join operators described in Section 4 were implemented as part of our own query engine and, alternatively, using the extensibility interfaces of the commercial database management system Oracle as outlined in Section 5. For evaluation purposes the latter implementation was used. The test environment was a PC system with a Pentium III (500 MHz) CPU running Linux and Oracle 8i. The extended operators and predicates were implemented using $\mathrm{C}++$. All test results refer to our implementation of the string similarity predicate based on the edit distance and supported by a trie index. A non-index implementation of the predicate is provided for comparison. Indexes are currently created on the fly and maintained in main memory only during operator processing time, which appears to be a reasonable approach considering the targeted data integration scenarios. The related performance impact is discussed below. 


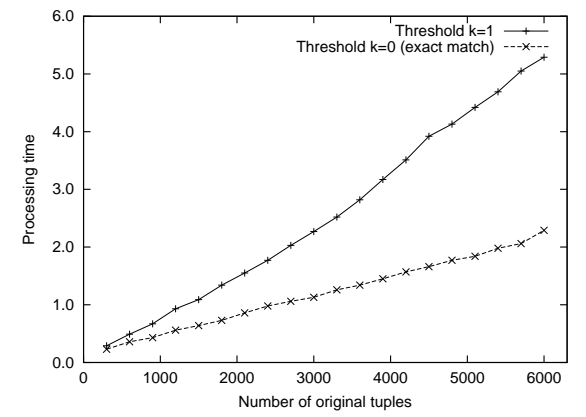

Fig. 1. Grouping with threshold $k=0$ and $k=1$

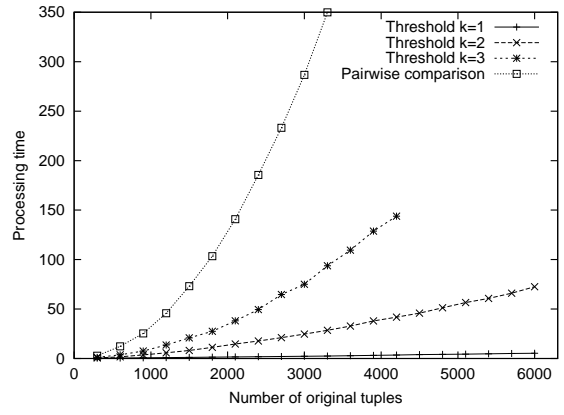

Fig. 2. Grouping with varying thresholds $k \geq 1$

For the grouping operator test runs separate data sets containing random strings were created according to the grade of similarity to be detected, i.e. for one original tuple between 0 and 3 copies were created that fulfilled the similarity condition of the test query. The test query consisted of an edit distance predicate on only one tuple. Using the edit distance with all operations having a fixed cost of 1 and a edit distance threshold $k$ on an attribute, each duplicate tuple had between 0 and $k$ deletions, insertions or transpositions. As the number of copies and the numbers of applied operations on the string attributes were equally distributed, for $n$ original tuples the total size of the data set to be processed was approximately $3 * n$ with an average distance of $\frac{k}{2}$ among the tuples to be detected as similar.

Grouping based on an exact matching $(k=0)$ has the expected complexity of $O(n)$, which results from the necessary iteration over the input set and the trie lookup in each step, which for an exact match requires average word-length comparisons, i.e. can be considered $O(1)$. This conforms to equality based grouping with hash table support. For a growing threshold the number of comparisons, i.e. the number of trie nodes to be visited, grows. This effect can be seen in Figure 1, where the complexity for $k=1$ appears to be somewhat worse than linear, but still reasonably efficient.

Actually, the complexity grows quickly for greater thresholds, as larger regions of the trie have to be covered. The dynamic programming approach of the similarity search ensures that even for the worst case each node is visited only once, which results in equal complexity as pairwise similarity comparison, not considering the cost for index maintenance etc. The currently used main memory implementation of the trie causes a constant overhead per insertion. Hence, the $O\left(n^{2}\right)$ represents the upper bound of the complexity for a rising threshold $k$, just like $O(n)$ is the lower bound. For growing thresholds the curve moves between these extremes with growing curvature. This is a very basic observation that applies to similarity based operations like similarity-based joins and selections as well, the latter providing the reason for these considerations having a complexity between $O(1)$ and $O(n)$. The corresponding test results are shown in Figure 2.

The previous test results were presented merely to make a general statement about the efficiency of the similarity-based grouping operator. An interesting question in real 


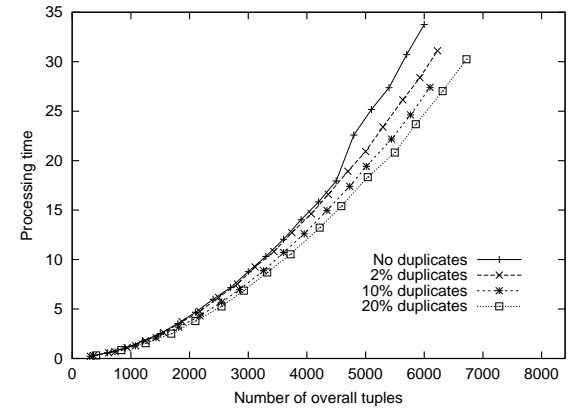

Fig. 3. Grouping with varying percentage of duplicates in the test data sets

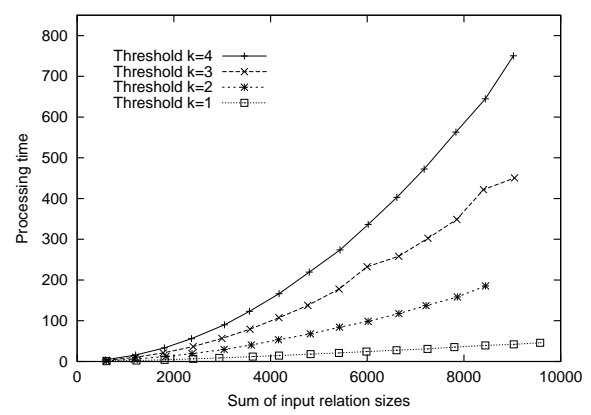

Fig. 4. Results for varying thresholds $k \geq 1$ for a similarity join

life scenarios would be, how the operator performs on varying ratios of duplicates in the tested data set. In Figure 3 the dependency between the percentage of duplicates and the required processing time is given for the threshold $k=2$. While the relative time complexity remains, the absolute processing time decreases for higher percentages of detectable duplicates. Obviously, and just as expected, using a similarity measure is more efficient, if there actually is similarity to detect. Otherwise, searching the trie along diverging paths represents an overhead that will not yield any results.

We received similar results for the described implementation of a similarity join. The test scenario consisted of two relations $R_{1}$ and $R_{2}$, with a random number of linked tuples, i.e. for each tuple in $R_{1}$ there were between 0 and 3 linked records in $R_{2}$ and the join attribute values were within a maximum edit distance. The results are shown in Figure 4. As the implementation of the join operation is similar to the grouping operation the complexity is between $O(n)$ and $O\left(n^{2}\right)$ depending on the edit distance threshold.

\section{Applications}

As described before, the problem of duplicate elimination in databases or during integration of various sources can be solved by applying the similarity-based grouping operations. Using an appropriate similarity predicate (see below for a discussion) potential redundant objects can be identified. However, applying a suitable similarity predicate is only the first step towards "clean" data: From each group of tuples a representative object has to be chosen. This merging or reconciliation step is usually performed in SQL using aggregate functions. But, in the simplest case of the builtin aggregates one is able only to compute minimum, maximum, average etc. from numeric values. As an enhancement modern DBMS provide support for user-defined aggregation functions (UDA) which allow to implement application-specific reconciliation functions. However, these UDAs are still too restricted for reconciliation because they support only one column as parameter. Here, the problem is to choose or compute a merged value from a set of possible discrepant values without looking at any other columns. We can 
mitigate this problem by allowing more than one parameter or by passing a structured value as parameter to the function.

In particular for reconciliation purposes we have defined a set of such enhanced aggregate functions including the following:

- pick_where_eq $(v, c O I)$ returns the value of column col of the first tuple, where the value of $v$ is true, i.e., $\neq 0$. In case of a group consisting of only one tuple, the value of this tuple is returned independently of the value of $V$.

- pick_where_min $(v, c O l)$ returns the value of column col of the tuple, where $v$ is minimal for the entire relation or group, respectively.

- pick_where_max $(v, c o l)$ returns the value of column col of the tuple, where $v$ is maximal.

- to_array ( $C O I$ ) produces an array containing all values from column col.

With the help of these functions several reconciliation policies can easily be implemented, one of them illustrated in the following example. We assume that the final value for column col of each group has to be taken from the tuple containing the most current date, which is represented as column m_date:

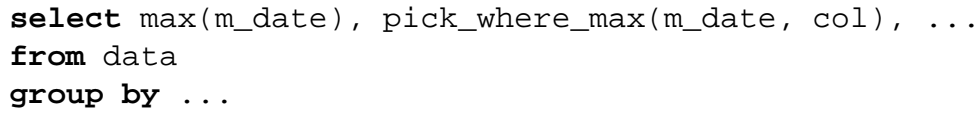

Another application-specific question is, how to specify the similarity predicate, consisting of the similarity or distance measure itself and the threshold. If the chosen threshold has such a major impact on the efficiency of similarity-based operations, as described in Section 6, the question is how to specify a threshold to meet requirements regarding efficiency and accuracy. Actually, this adds complexity to the well studied problem of over- and under-identification, i.e. falsely qualified duplicates. Information about the distance or similarity distribution can be used for deciding about a meaningful threshold, as well as for refining user-defined similarity predicates. Distance distributions usually conform to some natural distribution, according to the specific application, data types and semantics. Inconsistencies, such as duplicates, cause anomalies in the distribution, e.g. local minima or points of extreme curvature.

Figure 5(a) shows a result for a sample consisting of approximately 1.600 titles starting with an "E" from integrated sources of data on cultural assets. Nevertheless, drawing the conclusion of setting the edit distance threshold to receive a useful similarity predicate would lead to a great number of falsely identified tuples. For short titles there would be too many matches, and longer titles often do not match this way, because the length increases the number of typos etc.

Better results can be achieved by applying a relative edit distance $\operatorname{rdist}(x, y)=1-$ $\frac{\text { edist }(x, y)}{\max (x . \text { length }, \text {.length })}$ as a similarity measure as introduced in section 3 . The algorithm introduced in section 5 can easily be adjusted to this relative distance. Figure 5(b) shows the distribution of relative edit distances in the previously mentioned example relation. Using the first global minimum around 0.8 as a threshold, and analyzing matches in this area shows that it provides a good ratio of very few over- and under-identified tuples. A successive adjustment of similarity predicates using information from analytical data processing is also of interest for the creation of user-defined similarity predicates. 


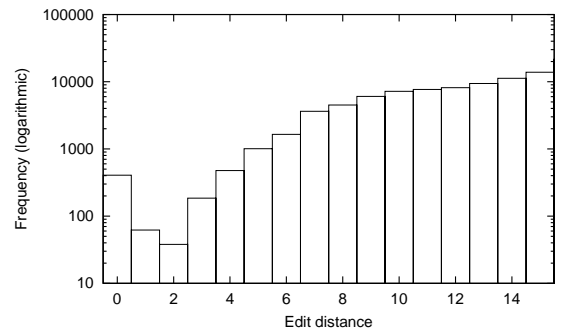

(a) Absolute edit distance distribution

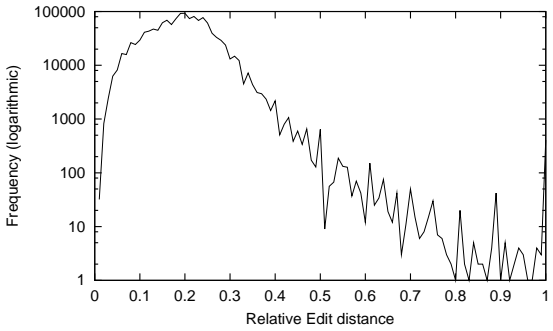

(b) Relative edit distance distribution

Fig. 5. Edit distance distributions in an integrated and sampled data set

\section{Conclusions}

In this paper we presented database operators for finding related data and identifying duplicates based on user-specific similarity criteria. The main application area of our work is the integration of heterogeneous data where the likelihood of occurrence of data objects representing related or the same real-world objects though containing discrepant values is rather high. Intended as an extended grouping operation and by combining it with aggregation functions for merging/reconciling groups of conflicting values our grouping operator fits well into the relational algebra framework and the SQL query processing model. In a similar way, an extended join operator takes similarity predicates used for both operators into consideration. These operators can be utilized in ad-hoc queries as part of more complex data integration and cleaning tasks.

Furthermore, we have shown that efficient implementations have to deal with specific index support depending on the applied similarity measure. For one of the most useful measures for string similarity (particularly for shorter strings) we have presented a trie-based implementation. The evaluation results illustrate the benefit of this approach even for relatively large datasets. Though we focused in this paper primarily on the edit distance measure, the algorithm for similarity grouping is able to exploit any kind of index support.

A still open issue is the question how to find and specify appropriate similarity criteria. In certain cases, basic similarity measures like the edit distance are probably not sufficient. As described in Section 3, application-specific similarity measures implementing domain heuristics (e.g. permutation of first name and last name) based on basic edit distances is often a viable approach. However, choosing the right thresholds and combinations of predicates during the design phase of an integrated system often requires several trial-and-error cycles. This process can be supported by analytical processing steps as shown in Section 7 and the corresponding tools. Such tools should allow an interactive investigation of analytical results as well corresponding samples from the data level, and are part of our information fusion workbench [4]. Providing the similarity-based operators as query primitives instead of dedicated application tools simplifies this and opens the opportunity for optimization. 


\section{References}

1. D. Calvanese, G. de Giacomo, M. Lenzerini, D. Nardi, and R. Rosati. A principled approach to data integration and reconciliation in data warehousing. In Proceedings of the International Workshop on Design and Management of Data Warehouses (DMDW'99), Heidelberg, Germany, 1999.

2. W. Cohen. Integration of heterogeneous databases without common domains using queries based on textual similarity. In L. M. Haas and A. Tiwary, editors, SIGMOD 1998, Proceedings ACM SIGMOD International Conference on Management of Data, June 2-4, 1998, Seattle, Washington, USA, pages 201-212. ACM Press, 1998.

3. D. Dey and S. Sarkar. A probabilistic relational model and algebra. ACM Transactions on Database Systems, 21(3):339-369, September 1996.

4. Oliver Dunemann, Ingolf Geist, Roland Jesse, Kai-Uwe Sattler, and Andreas Stephanik. A Database-Supported Workbench for Information Fusion: InFuse. In Christian S. Jensen, Keith G. Jeffery, Jaroslav Pokorný, Simonas Saltenis, Elisa Bertino, Klemens Böhm, and Matthias Jarke, editors, Advances in Database Technology - EDBT 2002, 8th International Conference on Extending Database Technology, Prague, Czech Republic, March 25-27, Proceedings, volume 2287 of Lecture Notes in Computer Science, pages 756 - 758. Springer, 2002.

5. R. Elmasri and S. B. Navathe. Fundamentals of Database Systems. Benjamin/Cummings, Redwood City, CA, 2 edition, 1994.

6. N. Fuhr. Probabilistic datalog - A logic for powerful retrieval methods. In Proceedings of the Eighteenth Annual International ACM SIGIR Conference on Research and Development in Information Retrieval, Retrieval Logic, pages 282-290, 1995.

7. H. Galhardas, D. Florescu, D. Shasha, and E. Simon. AJAX: an extensible data cleaning tool. In Weidong Chen, Jeffery Naughton, and Philip A. Bernstein, editors, Proceedings of the 2000 ACM SIGMOD International Conference on Management of Data, Dallas, Texas, volume 29(2), pages 590-590, 2000.

8. G. Graefe. Query Evaluation Techniques For Large Databases. ACM Computing Surveys, 25(2):73-170, 1993.

9. L. Gravano, P. G. Ipeirotis, H. V. Jagadish, N. Koudas, S. Muthukrishnan, and D. Srivastava. Approximate string joins in a database (almost) for free. In Proceedings of the 27th International Conference on Very Large Data Bases(VLDB '01), pages 491-500, Orlando, September 2001. Morgan Kaufmann.

10. J. M. Hellerstein, M. Stonebraker, and R. Caccia. Independent, Open Enterprise Data Integration. IEEE Data Engineering Bulletin, 22(1):43-49, 1999.

11. M. A. Hernández and S. J. Stolfo. The merge/purge problem for large databases. In Michael J. Carey and Donovan A. Schneider, editors, Proceedings of the 1995 ACM SIGMOD International Conference on Management of Data, pages 127-138, San Jose, California, 22-25 May 1995.

12. W. Kent. The breakdown of the information model in multi-database systems. SIGMOD Record, 20(4):10-15, December 1991.

13. Wen-Syan Li. Knowledge gathering and matching in heterogeneous databases. In AAAI Spring Symposium on Information Gathering, 1995.

14. E.-P. Lim, J. Srivastava, S. Prabhakar, and J. Richardson. Entity identification in database integration. In International Conference on Data Engineering, pages 294-301, Los Alamitos, Ca., USA, April 1993. IEEE Computer Society Press.

15. Sergio Luján-Mora and Manuel Palomar. Reducing Inconsistency in Integrating Data from Different Sources. In M. Adiba, C. Collet, and B.P. Desai, editors, Proc. of Int. Database Engineering and Applications Symposium (IDEAS 2001), pages 219-228, Grenoble, France, 2001. IEEE Computer Society. 
16. A. E. Monge and C. P. Elkan. The field matching problem: Algorithms and applications. In Evangelos Simoudis, Jia Wei Han, and Usama Fayyad, editors, Proceedings of the Second International Conference on Knowledge Discovery and Data Mining (KDD-96), page 267. AAAI Press, 1996.

17. A. E. Monge and C. P. Elkan. An efficient domain-independent algorithm for detecting approximately duplicate database records. In Proceedings of the Workshop on Research Issues on Data Mining and Knowledge Discovery (DMKD'97), 1997.

18. Gonzalo Navarro. A guided tour to approximate string matching. ACM Computing Surveys, 33(1):31-88, 2001

19. E. Schallehn, K. Sattler, and G. Saake. Extensible grouping and aggregation for data reconciliation. In Proc. 4th Int. Workshop on Engineering Federated Information Systems, EFIS'01, Berlin, Germany, 2001.

20. H. Shang and T. H. Merrett. Tries for approximate string matching. IEEE Transactions on Knowledge and Data Engineering, 8(4):540-547, 1996.

21. K. Shim, R. Srikant, and R. Agrawal. High-dimensional similarity joins. In Proceedings of the 13th International Conference on Data Engineering (ICDE'97), pages 301-313, Washington - Brussels - Tokyo, April 1997. IEEE.

22. F. Tseng, A. Chen, and W. Yang. A probabilistic approach to query processing in heterogeneous database systems. In Proceedings of the 2nd International Workshop on Research Issues on Data Engineering: Transaction and Query Processing, pages 176-183, 1992.

23. H. Wang and C. Zaniolo. Using sql to build new aggregates and extenders for object- relational systems. In A. El Abbadi, M.L. Brodie, S. Chakravarthy, U. Dayal, N. Kamel, G. Schlageter, and K.-Y. Whang, editors, Proc. of 26th Int. Conf. on Very Large Data Bases (VLDB'O0), Cairo, Egypt, pages 166-175. Morgan Kaufmann, 2000.

24. T. W. Yan and H. Garcia-Molina. Duplicate removal in information dissemination. In Proceedings of the 21st International Conference on Very Large Data Bases (VLDB '95), pages 66-77, San Francisco, Ca., USA, September 1995. Morgan Kaufmann Publishers, Inc.

25. G. Zhou, R. Hull, R. King, and J. Franchitti. Using object matching and materialization to integrate heterogeneous databases. In Proc. of 3rd Intl. Conf. on Cooperative Information Systems (CoopIS-95), Vienna, Austria, 1995. 